\title{
Teaching adaptive optics concepts in the high school classroom using an active engagement, experimental approach
}

Robert Sparks, Stephen Pompea, Constance Walker, Erin Dokter

Robert T. Sparks, Stephen M. Pompea, Constance E. Walker, Erin F. C. Dokter, "Teaching adaptive optics concepts in the high school classroom using an active engagement, experimental approach," Proc. SPIE 7783, Optics Education and Outreach, 77830C (30 August 2010); doi:

$10.1117 / 12.862633$

SPIE Event: SPIE Optical Engineering + Applications, 2010, San Diego, California, United States 


\title{
Teaching Adaptive Optics Concepts in the High School Classroom Using an Active Engagement, Experimental Approach
}

\author{
Robert T. Sparks ${ }^{\mathrm{a}}$, Stephen M. Pompea ${ }^{\mathrm{a}}$, Constance E. Walker ${ }^{\mathrm{a}}$, Erin F.C. Dokter ${ }^{\mathrm{b}}$ \\ ${ }^{a}$ National Optical Astronomy Observatory, 950 N. Cherry Ave, Tucson, AZ USA 85719 \\ ${ }^{\mathrm{b}}$ Office of Instruction and Assessment, The University of Arizona, 1500 E. University Boulevard, \\ Tucson AZ USA 85721
}

\begin{abstract}
Adaptive optics is rarely mentioned in high school physics classes due to its complex nature but is a valuable way to introduce key optics concepts that have practical applications. Over the last three years, we have been developing a series of hands-on activities targeted at high school students addressing various topics in adaptive optics. Using only high school math, these activities build a conceptual understanding of the processes involved in modern adaptive optics systems. The topics include atmospheric distortion, Shack-Hartmann sensors, and flexible mirrors. We will outline the activities as well as the results of classroom testing.
\end{abstract}

Keywords: Education, Adaptive Optics, High School

\section{INTRODUCTION}

Optics is a topic that is not always taught in depth at the high school level. Even advanced high school physics courses tend to focus on geometrical optics and basic image formation with little attention paid to more advanced topics in optics.Over the last few years, the Education and Public Outreach (EPO) group at the National Optical Astronomy Observatory (NOAO) have been developing a series of hands-on activities for high school students involving adaptive optics. The activities are being developed for the Giant Segmented Mirror Telescope (GSMT). These activities are based on our successful Hands-On Optics program.

The activities are packaged as a complete educational module. Each Adaptive Optics module contains all of the materials a teacher needs to do all of the activities with a class of students (except for standard supplies such as scissors). A comprehensive teacher's guide has background information, instructions on how to do all the activities, student worksheets and other resources.

\section{HANDS-ON OPTICS}

Hands-On Optics (HOO)[1] was funded by the National Science Foundation's (NSF) Informal Science Education division. The initial project ran from 2003-2007. HOO created a series of six modules each covering a different area of optics. These modules contained all the materials needed to do the activities with a classroom of students. HOO was targeted at informal venues such as after school science clubs and science centers. However, many teachers used HOO in the formal classroom as well.

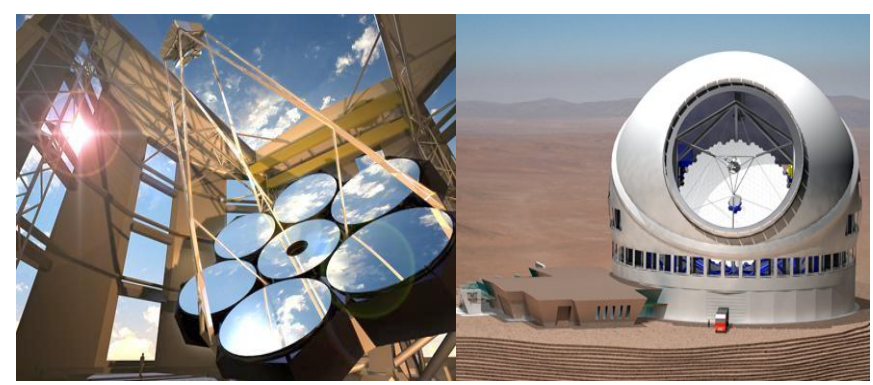

Two potential designs for the GSMT: The Giant Magellan Telescope (left) and the Thirty Meter Telescope (right). 
All of the activities were designed to correlate with national education standards. The manuals include information on the National Science Education Standards, the National Council of Teachers of Mathematics standards, and the the International Technology Education Association Standrds. HOO modules were originally targeted at middle school students. With modifications, the materials have been used with slightly younger students and older students up to community college students.

The Giant Segmented Mirror Telescope (GSMT) project provided a small grant to develop educational materials as part of their education efforts. We have developed anAdaptive Optics Module. Adaptive optics is a powerful way to educate students about applied optics and how the problem of atmospheric distortion of images can be addressed by the next generation of telescopes. We used out Hands-On Optics program as a starting point since we had already developed a module on lenses and image formation. Our goal was to extend the activities to include adaptive optics. We decided to use the HOO module diseign as a template for the Adaptive Optics module. A wide variety of activities and materials were tested to come up with the final design.

Extensive searching found few references to adaptive optics in high school textbooks. Astronomy 101 textbooks frequently have a section on adaptive optics at a very conceptual level. However, few hands-on activities involving adaptive optics exist at the high school level. The Center for Adaptive Optics (CFAO) at the University of California Santa Cruz have developed AO educational materials for college students. Unfortunately, the wavefront sensors and deformable mirrors used in their labs are cost prohibitive for many schools.

\section{THE ACTIVITIES}

The goal in the Adaptive Optics module it to trace the entire process from image formation to correcting the image using flexible mirrors. This allows students to understand each part of the process. We have taken a very conceptual rather than a mathematical approach in our design of the educational materials. Our kits were designed using a variety of low-cost materials.

Each activity in the manual is structured to help the teacher lead the class effectively. The activity has a complete list of materials needed for the activity as well as any classroom supplies the teachers should have handy (such as scissors). Learning goals are spelled out at the beginning of the activity. Complete directions on preparation are provided as well as instructions on leading the activity. Many of the activities have student handouts included. We have provided the teachers with background information on the scientific principles illustrated in each activity and ideas for going further.

The manual contains other information for the teacher including a summary of activities, all the National Standards that are covered by the activities, a glossary, and a "More Information for the Interested Educator section which goes into the topics more in depth.

The activities are described below. 


\subsection{Image Formation}

Image Formation focuses on how lenses can be used to focus light. Students measure the focal length of several different lenses by focusing light from a distant object. By using a distant object, the focal length and image distance are the same. The thin lens formula is discussed in the teacher's manual and it can be incorporated into the activity for more advanced students.

Students also explore other aspects of the image they form. The image will be inverted and reversed right to left. Two lenses made of identical types of glass are used which allow students to explore how lens shape affects the focal length of the lens. Due to the different focal lengths, the images have different sizes and brightnesses which can be discussed as well.

\subsection{It's Done With Mirrors}

This activity is very closely related to the Image Formation activity. Students are given a curved mirror and find the focal length of a curved mirror in a way that is similar to the Image Formation activity. The mirror is double sided so students can learn about the differences between convex and concave mirrors as well. This lesson helps students understand that images can be formed either by lens or mirrors.

\subsection{Build a Refracting Telescope}

In this activity students use the two lenses from Image Formation to build a small, low-power refracting telescope. Students learn which lens to use for the objective and which lens to use for the eyepiece as well as measure how far apart the lenses must be placed to produce a focused image. The image is upside down and students learn the function of each lens in a refracting telescope and why the image is inverted. A math extension teaches students how to calculate the magnification of a telescope. We have used this activity with the Project STAR refracting telescope kits as well as with the Galileoscope telescope kits.

An optional extension of this activity allows students to attempt to construct a reflecting telescope using the mirrors from the previous activity. Most modern telescopes are reflectors and this extension allows students to explore a different telescope design.

\subsection{Bubbles in the Air}

"Bubbles in the Air" gives the students an introduction to atmospheric distortion of images.

Students observe how a container of mineral oil distorts the image of an eye chart placed behind the mineral oil. Students can compare the pattern a laser makes when you shine it at a wall versus when you put a container of mineral oil in the path. The laser produces a nice round spot when you shine it at the wall but is spread out by the oil, illustrating how Earth's atmosphere distorts and spreads out starlight.

Another activity that illustrates the distortion process is a unique kinesthetic activity where students pretend to be a wavefront of light. Students stand in a straight line and lock elbows. They practice walking at the same speed to illustrate how light would travel in a vacuum.

To simulate the effects of Earth's atmosphere, the teacher lays out several poster boards on the ground. Some are red to indicate warmer areas and some are blue to represent colder areas (or they may have "hot" and "cold" written on them, preferably in red and blue markers respectively). As 
the students walk to simulate the movement of the wavefront, they are instructed to speed up when they walk across the "hot" areas and slow down when they walk across the "cold" areas. The end result is that the wavefront is distorted from its original shape due to these refractive effects caused by changes in the index of refraction for these different temperature areas.

\subsection{Tipping and Tilting}

Students begin to explore how to correct for atmospheric distortion in this activity. The simplest adaptive optics correction involves a tip-tilt mirror. Tip-tilt mirror systems have come down in price to the point where some amateur astronomers use tip-tilt modules such as the SBIG AO-8 or AO-L systems to help stabilize their images.

Our tip-tilt module uses a toy Labryinth game. Labyrinth is a common toy where the goal is to tip and tilt the board by using two knobs to guide a marble through a maze without the marble falling into a hole. We took the Labyrinth game and glued a mirror over the playing surface to create a model of a tip-tilt adaptive optics module.

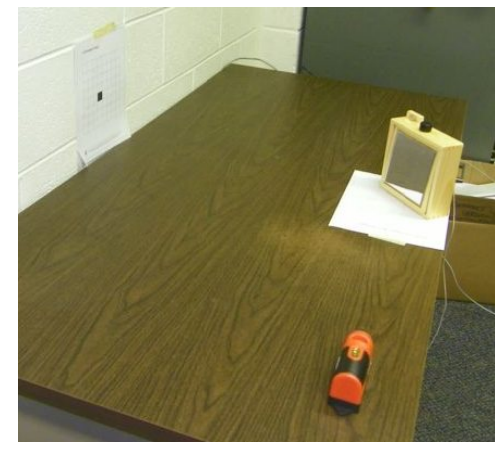

The tip-tilt mirror assembly and laser.

A grid is taped to the wall to simulate a CCD chip. A laser, representing a star, bounces off the mirror and lands on the grid at a particular pixel. As the laser is slowly moved to simulate atmospheric distortion, students learn which way they need to move the mirror to keep the star at a fixed pointing the CCD chip and avoid the blurring effects of the atmosphere. A worksheet helps students process their findings.

\subsection{The Shack-Hartmann Sensor}

This demonstration of a Shack-Hartmann(S-H) Sensor is based on work done at Carthage College (Arion, et al.). A S-H sensor is a lenslet array used to measure waveform deformation. The images from the lenses are projected onto a CCD chip. Based on the movement of the images, the distortion introduced by Earth's atmosphere can be measured. However, the lenslets in a S-H sensor are small and produce rapidly moving images that are impossible to see with the naked eye. A scaled up version of a $\mathrm{S}-\mathrm{H}$ sensor is necessary.

We made a version of the $\mathrm{S}-\mathrm{H}$ sensor demonstration using plastic

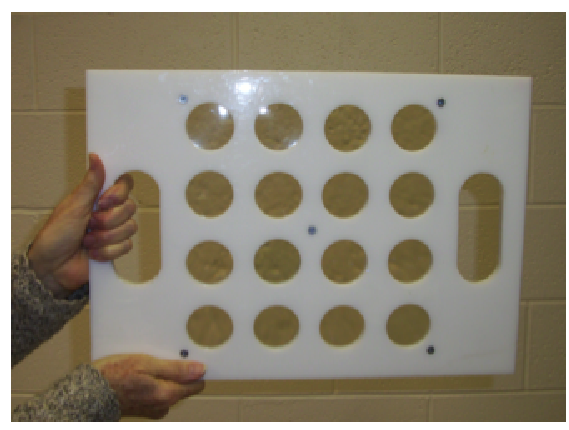

The Shack-Hartmann Sensor cut in a machine shop. 16 lenses of focal length $50 \mathrm{~cm}$ were assembled into a $4 \times 4$ array. A distant light source is shined at the H-S producing an array of 16 images on the wall. A container of mineral oil placed between the light source and the H-S can be used to simulate Earth's atmosphere and provide the distortion necessary to see the images move. 


\subsection{It's Done With Mirrors II}

This activity further explores how a curved mirror brings light to a focus. Students use a flexible piece of Mylar and a circular template to create a cross section of a spherical mirror. By shining three parallel lasers at the mirror, they can observe how the lasers converge at a point after bouncing off the mirror. We use laser levels for this experiment so students can clearly see the path of the lasers on a piece of paper placed on the table. The laser levels have a grating in front so they project a line onto the surface of the table.

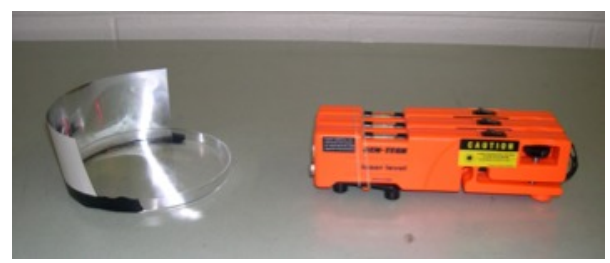

Exploring the focal length of a curved mirror.

Students can measure the focal length of the mirror and find the relationship between the radius of the mirror and its focal length.

\subsection{Closing the Loop: Flexing the Mirror}

The last step in the adaptive optics process is using a flexible mirror to correct for the distortions caused by Earth's atmosphere. The setup we used in the previous activity did not provide a good way to flex the mirror so another solution was needed.

We use Play-Doh to created a semi-circular backing for the mirror. The mirror starts out spherical and we place a piece of paper in front of the mirror with a mark to indicated the focal point of the mirror. When the lasers are parallel, all the beams should cross at the focal point. We keep the lasers toward the center of the mirror to avoid the complications added by spherical aberration.

By slightly turning the lasers so they are no longer parallel, you can simulate distortion by Earth's atmosphere. The lasers will not longer cross at the focal point. Students can use the Play-Doh to distort the shape of the mirror in an effort to get the three laser beams to cross at the focal point. When this is achieved, the image has been corrected.

We have experimented with other ways to create the distortion, but have not found a better solution that moving the lasers. Various types of Jell-O optics have been tried with limited success.

\subsection{Math Extensions: Resolution}

This section explores the concept of resolution. Resolution is defined and how to measure resolution is discussed. Teachers and students learn how to convert between degrees, radians, and arc seconds (the unit commonly used by astronomers).

Students participate in an activity where they can measure the height of another student using the small angle approximation. Students cut out an angle template the shows either a five or ten degree angle. Another student stands still. The first student moves closer or farther away until the size of the student is equal to the size of the angle. They measure the distance between them and use that information to find the height of the student. 


\section{CLASSROOM TESTING}

The activities were tested from October of 2009 through March of 2010 at a public high school in Tucson. The students were mostly labeled as learning disabled to various degrees. The school is located in an economically disadvantaged area of Tucson. The classroom teacher is a veteran teacher who has worked with the observatory in various education programs. The students had a wide range of abilities. A couple of the students were very interested in science and would consider a career in STEM fields. Other students had reading skills well below grade level. Several of the students showed a great deal of creativity when given open-ended problems to explore. Each week the students gave written feedback on the activities.

The teacher suggested adding Bell Work. Bell Work is a short written exercise at the beginning of the class period to help get the students focused on the lesson. We developed a series of Bell Work activities at high suggestion and noticed the students had improved behavior after we implemented these activities. The original order of the activities did not flow well from one to the other. We rearranged the activities to make a more logical progression in showing the students how adaptive optics works.

There were minor safety issues in the activities using lasers. We included a section on laser safety in the manual and every class in which we used lasers included a review of laser safety. Even with appropriate warnings, students would occasionally point lasers at each other. The lasers are low power class II lasers, but vigilance must be maintained for safety.

Most of the activities worked as expected although minor modifications needed to be made at various points. The mineral oil proved difficult to work with and got messier than anticipated. Enclosing the mineral oil in Ziploc bags would be a good idea to minimize the mess for the Bubbles in the Air section. We also found that you have to be careful what type of Mylar is used in the experiments. Thin Mylar did not make nearly as good mirrors as thicker Mylar.

Although the module was designed for use in informal education settings, the students focused much better in the activities with formal worksheets. This may be a result of the audience. We wrote worksheets for several of the activities that led to a guided inquirymodel.

The kinesthetic activity (part of Bubbles in the air) tested extremely well. Students took to it very quickly and spoke very highly of it in the written evaluations.

The students enjoyed when we could add real world examples to the activities. In early February of 2010, the U.S. Missile Defense Agency successfully tested the Airborne Defense Laser. The Airborne Defense Laser uses an adaptive optics system to keep the laser collimated allowing it to destroy its target. Students were very interested in applications such as the Airborne Defense Laser.

\section{FUTURE DISTRIBUTION OF THE KITS}

We have all of the materials for making kits and are in the process of final assembly of approximately 20 adaptive optics kits. We are looking for partners to test and use the kits in their 
programs. Some of the sites lined up for kits include the Center for Adaptive Optics, the HarvardSmithsonian Center for Astrophysics, the Gemini Observatory, Cerro-Tololo Inter-American Observatory, and the National Air and Space Museum.

We are currently exploring models for training the sites in use of the kits. Both in person workshops and online professional development workshops are being considered.

\section{REFERENCES}

[1] Pompea, S.M., Johnson, A., Arthurs E. and Walker, C.E, "Hands-On Optics: An Educational Initiative for Exploring Light and Color in After-School Programs, Museums, and Hands-On Science Centers", Proc. Ninth International Topical Meeting on Education and Training in Optics and Photonics, Marseille, France (2005)

[2] Arion, D.N., Crosby, K.M., Lyons, D., Rand, K. Randolph, A., "Hand-On Demonstration and Teaching Tools for Optics and Adaptive Optics", Proceedings of Education and Training In Optics and Photonics, 2003 